\title{
Quantitative Assessment of Air Trapping Using Inspiratory and Expiratory Low-Dose Computed Tomography
}

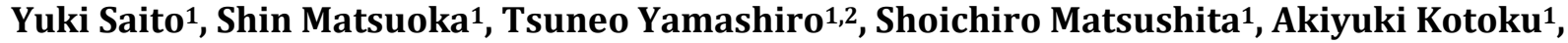 \\ Kunihiro Yagihashi', Hayato Tomita' ${ }^{1}$, Shingo Sakamoto' ${ }^{1}$, Shinji Saruya', Yasuo Nakajima1 \\ ${ }^{1}$ Department of Radiology, St. Marianna University School of Medicine, Kawasaki, Japan \\ ${ }^{2}$ Department of Radiology, Graduate School of Medical Science, University of the Ryukyus, Nishihara, Japan \\ Email:y3saito@marianna-u.ac.jp
}

Received 27 September 2015; accepted 1 December 2015; published 4 December 2015

Copyright (C) 2015 by authors and Scientific Research Publishing Inc.

This work is licensed under the Creative Commons Attribution International License (CC BY).

http://creativecommons.org/licenses/by/4.0/

(C) (i) Open Access

\begin{abstract}
Objective: The purpose of this study was to evaluate the effect of radiation dose reduction on the quantification of air trapping on expiratory CT. Materials and methods: This study was conducted as a retrospective evaluation of inspiratory and expiratory CT studies performed in routine clinical practice before and after alteration of the scanning protocol for expiratory CT at our institute. Eighty-six patients who had a clinical diagnosis of chronic obstructive pulmonary disease (COPD) and underwent inspiratory and expiratory CT and pulmonary function testing (PFT) were included. For the quantitative analysis, CT scans were obtained at six evenly spaced levels from the lung apices to the bases. The area of segmented lung without emphysema between -500 to -950 HU was obtained from the summation of six slices. The relative area between -900 and $-950 \mathrm{HU}$ for the area of the segmented lung (RA900-950) was calculated on both the inspiratory and expiratory scans. Comparisons of the RA-change between the standard-dose group $(200 \mathrm{~mA})$ and the low-dose group ( $80 \mathrm{~mA})$ were performed by Mann-Whitney $U$ test. Results: There was no significant difference between the standard-dose group and the low-dose group in the mean RA-change, and RA-change in both the standard-dose and low-dose groups correlated significantly with the results of PFT. In addition, there were no prominent differences in the correlation coefficients between the two groups. Conclusions: Low-dose CT could evaluate air trapping objectively and was not inferior to standard-dose CT for this purpose.
\end{abstract}

\section{Keywords}

Chronic Obstructive Pulmonary Disease, Emphysema, Air Trapping, Computed Tomography, Quantification 


\section{Introduction}

Air trapping is indicating the retention of excess gas in the lung of expiration, and is well known that it is correlated with pulmonary function. Expiratory computed tomography (CT) provides physiologic information concerning regional lung function, and has been used to reveal air trapping in patients with airway diseases such as small-airway disease, diffuse interstitial lung diseases. Chronic obstructive pulmonary disease (COPD) is characterized by the presence of airflow limitation [1] caused by small airway obstruction [2] [3]. Many studies have assessed the use of CT images in the quantitative analysis of airflow limitation in COPD. Because small airway disease cannot be visualized directly with current radiographic techniques, the extent of air trapping on expiratory CT, or paired inspiratory and expiratory CT scans can be used to evaluate small airway obstruction [4]-[11]. However, expiratory CT does expose patients to additional radiation, and reduction of radiation exposure is vital.

Bankier et al. evaluated the effect of radiation dose reduction on the visual quantification of air trapping on expiratory CT [6]. They found that the tube current-time product could be reduced from 80 to $20 \mathrm{mAs}$ without impairing the visual quantification of air trapping on expiratory CT. Practically, however, air trapping has been evaluated with objective quantitative methods using densitometric parameters such as the changes in relative percentage areas of certain attenuation values among entire lung volume between inspiratory and expiratory CT scans, or the ratio of the mean lung attenuation value of inspiration and expiration in previous studies [4] [7]-[10]. How the reduction of radiation exposure will affect those objective quantitative measurements has not been assessed. Thus, the purpose of this study is to evaluate the effect of radiation dose reduction on the quantification of air trapping on expiratory CT.

\section{Materials and Methods}

\subsection{Subjects}

This study was conducted as a retrospective evaluation of inspiratory and expiratory CT studies performed in routine clinical practice before and after alteration of the scanning protocol for expiratory CT at our institute. We changed tube current of the expiration CT into $80 \mathrm{~mA}$ from $200 \mathrm{~mA}$ from June, 2010. Reduction of tube current (mA) was used to reduce the radiation dose. The study was performed with institutional review board approval, and informed consent was waived.

Sixty-six patients who had a clinical diagnosis of COPD and underwent inspiratory and expiratory CT and pulmonary function testing (PFT), including 11 females and 55 males (mean age 75 years \pm 7 ; range 43 - 86 years), were included in this study. The standard-dose group of 26 patients (mean age $74 \pm 5$ years; range 59 82 years) was scanned with a $200-\mathrm{mA}$ protocol. The low-dose group after alteration of the scanning CT protocol of 40 patients (mean age $73 \pm 8$ years; range 43 - 86 years) was scanned with an 80 -mA protocol.

\subsection{CT Scanning}

All patients were scanned with a 64-detector CT scanner (Aquilion 64, Toshiba Medical Systems, Otawara, Tochigi, Japan). All patients were examined at full inspiration and full expiration in the supine position, without contrast material. The rotation time was 0.5 seconds, and the tube settings were $120 \mathrm{kVp}$ and $200 \mathrm{~mA}$ (standard-dose group) or $80 \mathrm{~mA}$ (low-dose group). Helical scanning was performed in the craniocaudal direction, with a beam pitch of 0.828 (helical pitch, 53). All images were reconstructed using a standard reconstruction algorithm with a slice thickness of $0.5 \mathrm{~mm}$ and a reconstruction interval of $0.5 \mathrm{~mm}$.

\subsection{Image Analysis}

For the quantitative analysis, CT scans were obtained at six evenly spaced levels from the lung apices to the bases. In general, scans were obtained at the following levels: above the aortic arch, between the aortic arch and the tracheal carina, at the tracheal carina, between the tracheal carina and the confluence of pulmonary veins, at the confluence of the pulmonary veins, and near the diaphragm. The inspiratory and expiratory CT images were transferred to a personal computer for quantitative analysis. For scans obtained at inspiration, special care was taken to define scan pairs with the highest possible agreement of the scan level using anatomic landmarks such as branching of bronchi. Each of the six CT images obtained at inspiration and expiration was segmented using a 
semiautomatic image-processing program (ImageJ Ver. 1.46, a public domain Java image processing program). Quantitative CT analysis of air trapping has been described elsewhere [7]. In brief, the following procedures were performed. The area of segmented lung without emphysema between -500 to $-950 \mathrm{HU}$ was obtained from the summation of six slices. The relative area between -900 and $-950 \mathrm{HU}$ for the area of the segmented lung (RA900-950) was calculated on both the inspiratory and expiratory scans as follows: RA900-950 (\%) = (segmented pixels between -900 to $-950 \mathrm{HU} /$ segmented pixel between -500 to $-950 \mathrm{HU}) \times 100(\%)$. The upper threshold value of $-900 \mathrm{HU}$ was chosen because this is the threshold value used for the detection of air trapping [12]. To evaluate the degree of air trapping in paired inspiratory/expiratory scans, the changes between inspiration and expiration in RA900-950 were calculated using the following formula: change of RA900-950 (RAchange $)=[($ inspiratory RA900-950 - expiratory RA900-950)/inspiratory RA900-950 $\times 100(\%)$.

\subsection{Pulmonary Function Tests}

PFT was performed within 2 weeks of obtaining CT scans. PFT including spirometry was performed. Forced expiratory volume in 1 second (FEV1) and forced vital capacity (FVC) were measured according to standard techniques, and the ratio of FEV1 to the forced vital capacity (FEV1/FVC) and mid-expiratory phase of the forced expiratory flow (FEF 25\% - 75\%) were obtained. Values for each pulmonary function test, except for FEV1/FVC, are expressed as percentages of predicted values.

\subsection{Statistical Analysis}

Statistical analysis was performed with commercially available software (JMP, version 9, SAS Institute, Cary, NC). Comparisons of the RA-change between the standard-dose group and the low-dose group were performed by the Mann-Whitney U test. Relationships between RA-change and the PFT results of each group were evaluated by the Spearman's rank correlation analysis. Linear regression analyses were performed to evaluate the relationships between RA-change and the PFT results of each group by the Spearman's rank correlation coefficient.

\section{Results}

There was no significant difference $(p=0.298)$ in RA-changes between the standard-dose group and the low-dose group. The correlation between the measured CT parameter and the PFT results of each group are shown in Table 1. RA-change in both the standard-dose and the low-dose group correlated significantly with the PFT results. In addition, there were no prominent differences in the correlation coefficients between the two groups.

\section{Discussion}

In this study, we found that objective quantitative CT evaluation of air trapping was possible with low-dose CT. This result was consistent with the findings of a previous study that examined air trapping visually on expiratory low dose CT [6].

Radiation dose reduction is a practical issue in CT quantitation. In the quantitative evaluation of the extent of emphysema, several investigations have been conducted. Yuan et al. evaluated the effect of radiation dose on quantitative CT scan measurements of emphysema in smokers [13], and they concluded that the change in radiation dose significantly affected measurements of emphysema assessed using mean lung density, threshold, or percentile methods. In contrast, Gierada et al. compared standard- and low-radiation-dose techniques in the CT quantification of emphysema [14], and they confirmed that low-dose technique had a minimal effect on CT

Table 1. The correlation between the measured CT parameter and the results of PFTs.

\begin{tabular}{cccccccc}
\hline \multirow{2}{*}{ Table Head } & \multicolumn{2}{c}{ FEF $25 \%-75 \%$} & \multicolumn{2}{c}{ FEV1/FVC } & \multicolumn{2}{c}{ FEV 1\% Predicted } \\
\cline { 2 - 7 } & $\rho$ & $P$ & $\rho$ & $P$ & $\rho$ & $P$ \\
\hline Low-dose group (80 mA) & 0.6854 & $<0.0001$ & 0.7799 & $<0.0001$ & 0.7456 & $<0.0001$ \\
Standard-dose group (200 mA) & 0.5095 & 0.0078 & 0.6109 & 0.0009 & 0.4619 & 0.0175 \\
\hline
\end{tabular}


quantification of emphysema. In regards to the quantification of emphysema, the effect of using low-dose technique has been controversial. Meanwhile, despite using the same densitometry technique to evaluate air trapping, no significant differences are found between standard- and low-dose evaluations. From this present study, we cannot explain these results and differences with the quantitative evaluation of emphysema, but the different attenuation threshold values being used may be related to the differences between quantitative evaluation of air trapping and emphysema.

The disadvantage of low-dose settings is the increase in image noise. Boone et al. evaluated the relationship between image noise and radiation dose reduction in CT, and they reported that image noise increases at lower $\mathrm{mA}$ and lower $\mathrm{kVp}$ settings [15]. Since image noise is not evaluated in this study, it can be possible that image noise affects the quantification of air trapping. However, there are no significant differences in the relationships with the PFT results between standard- and low-dose techniques. Thus, image noise in low-dose scans appears to have a minimal effect on CT quantification of air trapping. In general, expiratory scans are not used for visual evaluation clinically.

In conclusion, the results of the present study confirm that objective quantification of the extent of air trapping is possible with low-dose technique. To reduce radiation exposure, low-dose technique should be adopted in the quantitative evaluation of air trapping. But this present study has several limitations. First, the sample size of this study is relatively small, and this is a retrospective analysis. To confirm the present results, a prospective analysis with a large patient sample should be conducted. Second, the evaluation of the relationships with the PFT results is not sufficient. In most patients, measurements of lung volume parameters including functional residual capacity, residual volume, and total lung capacity are not performed. However, small airway obstruction can be evaluated with FEF 25\% - 75\% to some degree. Third, CT parameters are measured in six slices. Quantitative analysis with three-dimensional reconstruction images using a multi-slice CT scanner should be considered for future studies because misregistration of CT slices between inspiration and expiration may have affected the present results.

\section{Conflict of Interest}

None of the authors have any conflict of interests to disclose.

\section{References}

[1] Pauwels, R.A., Buist, A.S., Calverley, P.M., Jenkins, C.R. and Hurd, S.S., GOLD Scientific Committee (2001) Global Strategy for the Diagnosis, Management, and Prevention of Chronic Obstructive Pulmonary Disease. NHLBI/WHO Global Initiative for Chronic Obstructive Lung Disease (GOLD) Workshop Summary. American Journal of Respiratory and Critical Care Medicine, 163, 1256-1276. http://dx.doi.org/10.1164/ajrccm.163.5.2101039

[2] Hogg, J.C., Macklem, P.T. and Thurlbeck, W.M. (1968) Site and Nature of Airway Obstruction in Chronic Obstructive Lung Disease. The New England Journal of Medicine, 278, 1355-1360. http://dx.doi.org/10.1056/NEJM196806202782501

[3] Yanai, M., Sekizawa, K., Ohrui, T., Sasaki, H. and Takishima, T. (1992) Site of Airway Obstruction in Pulmonary Disease: Direct Measurement of Intrabronchial Pressure. Journal of Applied Physiology, 72, 1016-1023.

[4] Gevenois, P.A., De Vuyst, P., Sy, M., Scillia, P., Chaminade, L., de Maertelaer, V., et al. (1996) Pulmonary Emphysema: Quantitative CT during Expiration. Radiology, 199, 825-829. http://dx.doi.org/10.1148/radiology.199.3.8638012

[5] Eda, S., Kubo, K., Fujimoto, K., Matsuzawa, Y., Sekiguchi, M. and Sakai, F. (1997) The Relations between Expiratory Chest CT Using Helical CT and Pulmonary Function Tests in Emphysema. American Journal of Respiratory and Critical Care Medicine, 155, 1290-1294. http://dx.doi.org/10.1164/ajrccm.155.4.9105069

[6] Bankier, A.A., Schaefer-Prokop, C., De Maertelaer, V., Tack, D., Jaksch, P., Klepetko, W., et al. (2007) Air Trapping: Comparison of Standard-Dose and Simulated Low-Dose Thin-Section CT Techniques. Radiology, 242, 898-906. http://dx.doi.org/10.1148/radiol.2423060196

[7] Matsuoka, S., Kurihara, Y., Yagihashi, K. and Nakajima, Y. (2007) Quantitative Assessment of Peripheral Airway Obstruction on Paired Expiratory/Inspiratory Thin-Section Computed Tomography in Chronic Obstructive Pulmonary Disease with Emphysema. Journal of Computer Assisted Tomography, 31, 384-389. http://dx.doi.org/10.1097/01.rct.0000243457.00437.10

[8] Matsuoka, S., Kurihara, Y., Yagihashi, K., Hoshino, M., Watanabe, N. and Nakajima, Y. (2008) Quantitative Assessment of Air Trapping in Chronic Obstructive Pulmonary Disease Using Inspiratory and Expiratory Volumetric MDCT. AJR. American Journal of Roentgenology, 190, 762-769. http://dx.doi.org/10.2214/AJR.07.2820 
[9] Busacker, A., Newell Jr., J.D., Keefe, T., Hoffman, E.A., Granroth, J.C., Castro, M., et al. (2009) A Multivariate Analysis of Risk Factors for the Air-Trapping Asthmatic Phenotype as Measured by Quantitative CT Analysis. Chest, 135, 48-56. http://dx.doi.org/10.1378/chest.08-0049

[10] Matsuoka, S., Yamashiro, T., Washko, G.R., Kurihara, Y., Nakajima, Y. and Hatabu, H. (2010) Quantitative CT Assessment of Chronic Obstructive Pulmonary Disease. Radiographics: A Review Publication of the Radiological Society of North America, 30, 55-66. http://dx.doi.org/10.1148/rg.301095110

[11] Boiselle, P.M., Litmanovich, D.E., Michaud, G., Roberts, D.H., Loring, S.H., Womble, H.M., et al. (2013) Dynamic Expiratory Tracheal Collapse in Morbidly Obese COPD Patients. Copd, 10, 604-610. http://dx.doi.org/10.3109/15412555.2013.781149

[12] Newman, K.B., Lynch, D.A., Newman, L.S., Ellegood, D. and Newell Jr., J.D. (1994) Quantitative Computed Tomography Detects Air Trapping Due to Asthma. Chest, 106, 105-109. http://dx.doi.org/10.1378/chest.106.1.105

[13] Yuan, R., Mayo, J.R., Hogg, J.C., Pare, P.D., McWilliams, A.M., Lam, S., et al. (2007) The Effects of Radiation Dose and CT Manufacturer on Measurements of Lung Densitometry. Chest, 132, 617-623. http://dx.doi.org/10.1378/chest.06-2325

[14] Gierada, D.S., Pilgram, T.K., Whiting, B.R., Hong, C., Bierhals, A.J., Kim, J.H., et al. (2007) Comparison of Standardand Low-Radiation-Dose CT for Quantification of Emphysema. American Journal of Roentgenology, 188, 42-47. http://dx.doi.org/10.2214/AJR.05.1498

[15] Boone, J.M., Geraghty, E.M., Seibert, J.A. and Wootton-Gorges, S.L. (2003) Dose Reduction in Pediatric CT: A Rational Approach. Radiology, 228, 352-360. http://dx.doi.org/10.1148/radiol.2282020471 